\title{
The metallic state of the organic conductor TMTSF-DMTCNQ at low temperature under pressure $(*)(* *)$
}

\author{
A. Andrieux, C. Duroure, D. Jérome \\ Laboratoire de Physique des Solides, Université Paris-Sud, 91405 Orsay, France \\ and $\mathrm{K}$. Bechgaard \\ H. C. Oersted Institute, Universitetsparken 5, DK 2100, Copenhagen, Denmark
}

(Reçu le $1^{\text {er }}$ juin 1979, accepté le 14 juin 1979)

\begin{abstract}
Résumé. - Nous présentons la mise en évidence d'un état de conductivité élevée $\left(\sigma>10^{5}(\Omega \mathrm{cm})^{-1}\right)$ à basse température, stabilisé sous haute pression $(P>10 \mathrm{kbar})$ dans le conducteur organique quasi unidimensionnel TMTSF-DMTCNQ. L'étude de la magnétorésistance suggère que parmi plusieurs interprétations de l'état métallique l'hypothèse d'une supraconductibilité quasi unidimensionnelle de type BCS persistant jusqu'à $30 \mathrm{~K}$ environ pourrait être retenue.
\end{abstract}

\begin{abstract}
We present experimental evidences for a high conductivity state $\left(\sigma>10^{5}(\Omega . \mathrm{cm})^{-1}\right)$ stabilized by high pressure $(P>10 \mathrm{kbar})$ at low temperature in the quasi one-dimensional organic conductor TMTSFDMTCNQ. The magnetoresistance investigations suggest among several possible interpretations of the metallic state, the existence of a quasi 1-D BCS-like superconductivity remaining up to $\approx 30 \mathrm{~K}$.
\end{abstract}

This note presents the finding in an organic conductor of a metallic-like high conductivity state stable under high pressure down to $1.2 \mathrm{~K}$ : the charge transfer compound TMTSF-DMTCNQ (tetramethyl tetraselenafulvalene-dimethyl tetracyanoquinodimethane).

The TMTSF-DMTCNQ structure [1,2] exhibits the usual features of the TTF-TCNQ family, namely, the existence of segregated and uniform stacks of TMTSF and DMTCNQ molecules. Consequently, this compound presents pronounced quasi 1-D conductivity properties.

A sharp metal to insulator phase transition at $42 \mathrm{~K}$ has been revealed by transport properties [3]. As shown by ESR susceptibility [4] the acceptor chain (DMTCNQ) electron bandwidth is likely to be rather small. Moreover the pronounced 1-D character is suggested by both the crystal structure [2] and the narrowness of the ESR line [4].

$\left(^{*}\right)$ La version française de cet article paraîtra dans le $n^{\circ} 20$ des C.R.A.S. du 28 mai 1979.

(**) This work has been supported in part by the DGRST contract $\mathrm{n}^{\circ}$ 78-7-0313.
Preliminary diffuse $X$-ray diffraction patterns show that the bands are $1 / 4$ field [5], namely, a 0.5 charge transfer and a 4th order commensurability under atmospheric pressure. Consequently, the Fermi level in TMTSF-DMTCNQ lies closer to the band edge Van-Hove singularity than in TTF-TCNQ or in any other two-conducting chains compounds known up to date.

The transport properties under pressure have been looked at with the purpose of studying the influence of commensurability both on the phase diagram [6] and on the conductivity mechanism [7]. The goal was also the possibility of stabilization of a metallic state at low temperature under high pressure [8].

The longitudinal conductivity has been measured with the current techniques in our laboratory, on 29 single crystals of about $1 \times 0.05 \times 0.02 \mathrm{~mm}^{3}$ in size. At $300 \mathrm{~K}$ the conductivity ranges around $700(\Omega . \mathrm{cm})^{-1}$.

The phase transition temperature is only very weakly pressure dependent up to $\simeq 10 \mathrm{kbar}$ and drops sharply down to at least $1.2 \mathrm{~K}$ for all higher pressures. In the latter pressure domain the sample resistance exhibits a metallic character from ambient tempera- 
ture down to liquid helium temperature. Figures $1 a, b$ display the resistance behaviour for two samples at $P=13$ kbar.

In most samples the $4.2 \mathrm{~K}$ conductivity under pressure is bigger than $10^{5}(\Omega . \mathrm{cm})^{-1}$ and can eventually reach $2 \times 10^{5}(\Omega . \mathrm{cm})^{-1}$ in some cases. A

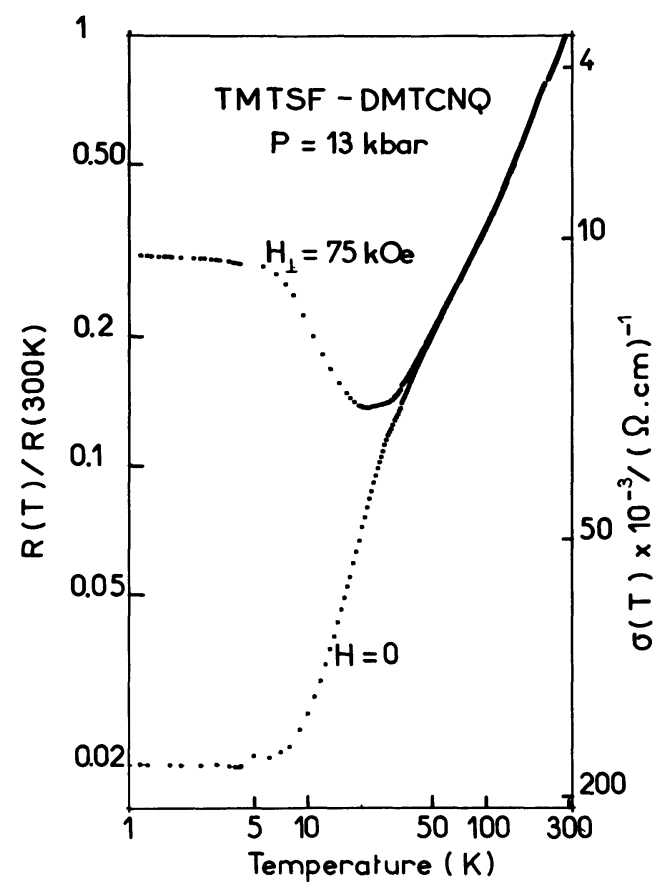

a)

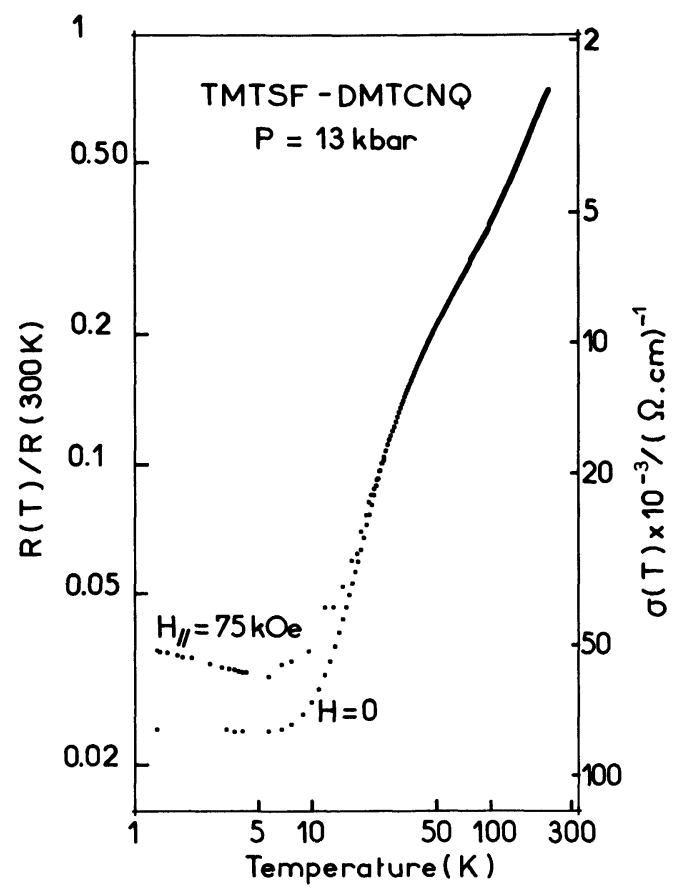

b)

Fig. 1. - Temperature dependence of the longitudinal resistivity of 2 different samples of TMTSF-DMTCNQ at $13 \mathrm{kbar}: a) H=0$ and $\left.H_{\perp}=75 \mathrm{kOe}, b\right) \quad H=0$ and $H_{\|}=75 \mathrm{kOe}$. remarkable effect has been noticed with the application of a magnetic field perpendicular to the large conductivity axis of the sample, figure $1 a$. Under magnetic field $(75 \mathrm{kOe})$, the temperature dependence of the resistivity shows the existence of a shallow metal to insulator transition around $12 \mathrm{~K}$.

This behaviour is reminiscent of the case of HMTSFTCNQ at atmospheric pressure, for which a structural phase transition at $24 \mathrm{~K}$ has been found to accompany the metal to insulator transition $[8,9]$.

The influence of a $75 \mathrm{kOe}$ magnetic field parallel to the chains in far weaker as shown on figure $1 b$. Crudely speaking, it does not modify significantly the general trends of the resistance temperature dependence. Since the angle between the magnetic field and the chain axis cannot be zeroed with an accuracy better than $\simeq 5^{\circ}$, the weak magnetoresistance effect, figure $1 b$, may be due to misalignement.

The stability of a metallic state at $1.2 \mathrm{~K}$ in a quasi 1-D conductor raises some serious problems. We shall suggest some possible interpretations for the results of figure 1 later in this note.

It has been shown that a large interchain tunnelling coupling can at low temperature stabilize a metallic state instead of the insulating Peierls state [10]. This effect has actually been observed in impure HMTSFTCNQ samples [8] under pressure. However, the conductivity of the metallic state thus stabilized does not grow significantly above $10^{4}(\Omega . \mathrm{cm})^{-1}$. Moreover, the temperature dependence of the HMTSFTCNQ resistivity at $P>4 \mathrm{kbar}$ is not modified by the application of a transverse magnetic field of $28 \mathrm{kOe}$ [11]. Most electronic properties of HMTSFTCNQ have been satisfyingly explained with a semimetallic band model [12].

Incommensurate CDW's can also contribute significantly to the electronic conduction of 1-D conductors [13].

For TTF-TCNQ this has been proved thanks to the measurements of the longitudinal [7] and transverse [14] conductivity in the vicinity of the $\times 3$ commensurability under pressure.

However the collective Fröhlich fluctuating contribution is proportional to the CDW coherence length [13] and its rise towards low temperature is limited by the interchain Coulomb pinning mechanism leading to an insulating state [15]. As far as TTF-TCNQ is concerned, the conductivity maximum of Fröhlich-like origin reaches $\simeq 2 \times 10^{4}(\Omega . \mathrm{cm})^{-1}$ at $60 \mathrm{~K}$. This value is nevertheless 10 times smaller than the value $\sigma(4.2 \mathrm{~K})$ in TMTSF-DMTCNQ, figure $1 a$.

The last possibility which we shall investigate has not been observed up to now. The existence of a superconducting BCS-like state could explain both the large values found for $\sigma(4.2 \mathrm{~K})$ and the influence of a magnetic field. With such a picture the superconducting transition can conceivably be substantially broadened by the pronounced 1-D character of the conduc- 
tor $[2,4]$. The growth of the conductivity below $4.2 \mathrm{~K}$ might possibly be limited by some breaks in the chains which are always present on a macroscopic scale in imperfect crystals, as observed in superconducting $\mathrm{SN}_{x}$ [16].

Considering the curvature of the resistivity temperature dependence between 40 and $10 \mathrm{~K}$, figure 1 , the resistivity might possibly reach a zero value below, say, $10 \mathrm{~K}$ in perfect samples, if real 3-D ordering takes place.

The interchain coupling, necessary for the establishment of a superconducting state in charge transfer compounds, is not negligible as shown in TTFTCNQ by the 3-D behaviour of the elastic coefficients [17]. Consequently, the superconducting transition temperature might be higher than $10 \mathrm{~K}$.

Assuming the model of an anisotropic type II superconductor (used for $\mathrm{SN}_{x}$ [18]) to be valid for TMTSF-DMTCNQ, a $75 \mathrm{kOe}$ transverse field is presumably larger than $H_{\mathrm{c}_{2}}^{\perp}$, and therefore breaks the superconducting pairs. This pair-breaking effect allows the establishment of a low temperature Peierls state [19], figure 1a. Under transverse magnetic field, the conductivity reaches $\simeq 3 \times 10^{4}(\Omega . \mathrm{cm})^{-1}$ around $22 \mathrm{~K}$, figure $1 a$.

Preliminary studies of the pressure dependence of the conductivity at $T>42 \mathrm{~K}$ and of the transverse conductivity suggest a collective Fröhlich fluctuating origin for the conductivity at high temperature, say
$T \gtrsim 42 \mathrm{~K}$. Thus we are tempted to believe that under strong transverse magnetic field the large value reached by the conductivity at $22 \mathrm{~K}$ is due to the fluctuating Fröhlich contribution. The only weak effect of a parallel field on the resistance indicates the existence of a large value for $H_{\mathrm{c}_{2}}^{\|}$(this value may even be larger than the paramagnetic limit of $\simeq 200 \mathrm{kDe}$, for $\left.T_{\mathrm{c}} \simeq 10 \mathrm{~K}\right)$.

Finally, the proximity between the Fermi level and a Van-Hove singularity [20] on the one hand and a weaker covalency interchain effect as compared to the neighbouring compounds TSF-TCNQ or TTF-TCNQ on the other hand might increase the superconducting transition temperature.

In conclusion, this work has conclusively established the existence of a very high conductivity state of metallic character in TMTSF-DMTCNQ under pressure. We have suggested among several explanations, the possibility of a quasi 1-D BCS-like mechanism contributing to the conductivity below $30 \mathrm{~K}$. More high pressure studies are in progress : ac susceptibility, thermoelectric power and tunnelling.

Acknowledgments. - We thank P. Chaikin, J. Friedel, W. Kohn, H. J. Schulz for numerous and fruitful discussions, and J. P. Pouget for his help with the preliminary X-ray diffuse scattering experiments.

We thank J. R. Andersen for his help in the sample preparation.

\section{References}

[1] Andersen, J. R., Jacobsen, C. S., Rindorf, G., Soling, H. and BechgaArd, K., J. Chem. Soc. Chem. Commun. (1975) 883-884.

[2] Andersen, J. R., BechgaARd, K., JACobsen, C. S., Rindorf, G., Soling, H. and ThORup, N., Acta Crystallogr. B 34 (1978) 1901-1905.

[3] Jacobsen, C. S., Mortensen, K., Andersen, J. R., BeChGAARD, K., Phys. Rev. B 18 (1978) 905-921.

[4] Tomkiewicz, Y., Andersen, J. R., Taranko, A. R., Phys. Rev. B 17 (1978) 1579-1591.

[5] Pouget, J. P., Private communication. It is commensurable at $P=0 \mathrm{kbar}$.

[6] Friend, R. H., Miljak, M., Jérome, D., Phys. Rev. Lett. 40 (1978) 1048

[7] Andrieux, A., Schulz, H. J., Jérome, D. and BechgaARD, K., Phys. Rev. Lett., submitted in 1979.

[8] JÉrome, D. and Weger, M., Chemistry and Physics of OneDimensional Metals, H. J. Keller (Plenum-Press, New York) 1977

[9] Non published results, obtained with high purity samples. MilJAK, M., COOPER, J. R., JÉROME, D. and BeChGAARD, K. and also Pouget, J. P., Private communication.

[10] Horovitz, B., Gutfreund, H., Weger, M., Phys. Rev. B 12 (1975) 3174-3185.

[11] Cooper, J. R., Weger, M., Delplanque, G., Jérome, D. and BechgaArd, K., J. Physique Lett. 37 (1976) L-349. 353 .
[12] Weger, M., Solid State Commun. 19 (1976) 1149-1155.

[13] Allender, D., Bray, J. W. and Bardeen, J., Phys. Rev. B 9 (1974) 119-129.

Patton, B. R. and Sham, L. J., Phys. Rev. Lett. 33 (1974) 638-641.

RICE, M. J., Solid State Commun. 16 (1975) 1285-1288.

[14] Andrieux, A., Schulz, H. J., Jérome, D. and BechgaArd, K., J. Physique Lett., to be published in 1979.

[15] Heeger, A. J., Chemistry and Physics of One-Dimensional Metals, H. J. Keller editor (Plenum Press, New York) 1977.

[16] Greene, R. L., Street, G. B. and Suter, L. J., Phys. Rev. Lett. 34 (1975) 577-579.

[17] Debray, D., Millet, R., Jérome, D., Barišić, S., Giral, L. and FABre, J. M., J. Physique Lett. 38 (1977) L-227-231.

[18] Azevedo, L. J., Clark, W. G., Deutscher, G., Greene, R. L., Street, G. B., Suter, L. J., Solid State Commun. 19 (1976) 197-201.

[19] Bichkov, Yu. A., Gorkov, L. P. and Dzyaloshinskin, I. E., Sov. Phys. JETP 23 (1966) 489-501.

Gutfreund, H., Horovitz, B., Weger, M., J. Phys. $C$ (1974) 383-388.

Levin, K., Mills, D. L. and Cunningham, S. L., Phys. Rev. B 10 (1974) 3821-3843.

Horovitz, B., Phys. Rev. B 16 (1977) 3943-3954.

[20] Labbé, J., Barišıć, S. and Friedel, J., Phys. Rev. Lett. 19 (1967) 1039-1041. 